\title{
Eastern Staining: A Simple Recombinant Protein Detection Technology Using a Small Peptide Tag and Its Counter Partner Which is a Fluorescent Compound
}

\author{
Jae-Jung Lee ${ }^{1, *}$, Jun-Young Kim ${ }^{2, *}$, Duanting Zhai ${ }^{2}$, Seong-Wook Yun' and Young-Tae Chang ${ }^{1,2, *}$ \\ 'Laboratory of Bioimaging Probe Development, Singapore Bioimaging Consortium, Agency for Science, Technology and Research, Biopolis, Singapore \\ ${ }^{2}$ Department of Chemistry, MedChem Program of Life Sciences Institute, National University of Singapore, Singapore \\ ${ }^{\dagger}$ These authors contributed equally to this work
}

\section{Subject areas; Bioimaging}

Author contribution; J-J.L. did molecular cloning, biological experiments and wrote manuscripts; J-Y.K. also did molecular biological works such as optimization for expression, Western blot analysis; D.Z. made BDED compound; Y.T.J. has supervised to the authors.

*Correspondence and requests for materials should be addressed to Y.T.J. (chmcyt@nus.edu. $\mathrm{sg})$.

Editor; Hyun Joo, Inje University, Korea

Received June 01, 2012

Accepted June 12, 2012

Published June 15, 2012

Citation; Lee, J-J., et al. Eastern Staining: A Simple Recombinant Protein Detection Technology Using a Small Peptide Tag and Its Orthogonal Fluorescent Compound. IBC 2012, 4:5, 1-9. doi: 10.4051/ibc.2012.4.2. 0005

Funding; This work was supported by the intramural funding from the A*STAR Biomedical Research Council and a Singapore Ministry of Education Academic Research Fund Tier 2 (MOE2010-T2-1-025).

Competing interest; All authors declare no financial or personal conflict that could inappropriately bias their experiments or writing.
() Lee, $\mathrm{J}-\mathrm{J}$, et al. This is an Open Access article distributed under the terms of the Creative Commons Attribution Non-Commercial License (http://creativecommons.org/licenses/by-nc/3.0) which permits unrestricted non-commercial use, distribution, and reproduction in any medium, provided the original work is properly cited.

\section{SYNOPSIS}

Small peptide tags such as c-myc, HA, or FLAG tag have facilitated efficient Westernblotting of proteins of interest especially when specific antibodies for the proteins are not available. However, the conventional Western-blotting requires the multi-steps process taking at least several hours up to two days. With examples of various applications, here we show a convenient and time-saving method for protein detection which employs a fluorescent chemical BDED and its binding peptide RC-tag. And we propose "Estern staining", as a standard term for protein detection method using fluorescent chemicals and their binding small peptide tags. Eastern staining may substitutes for the time-consuming "immuno-staining" in many versatile applications.

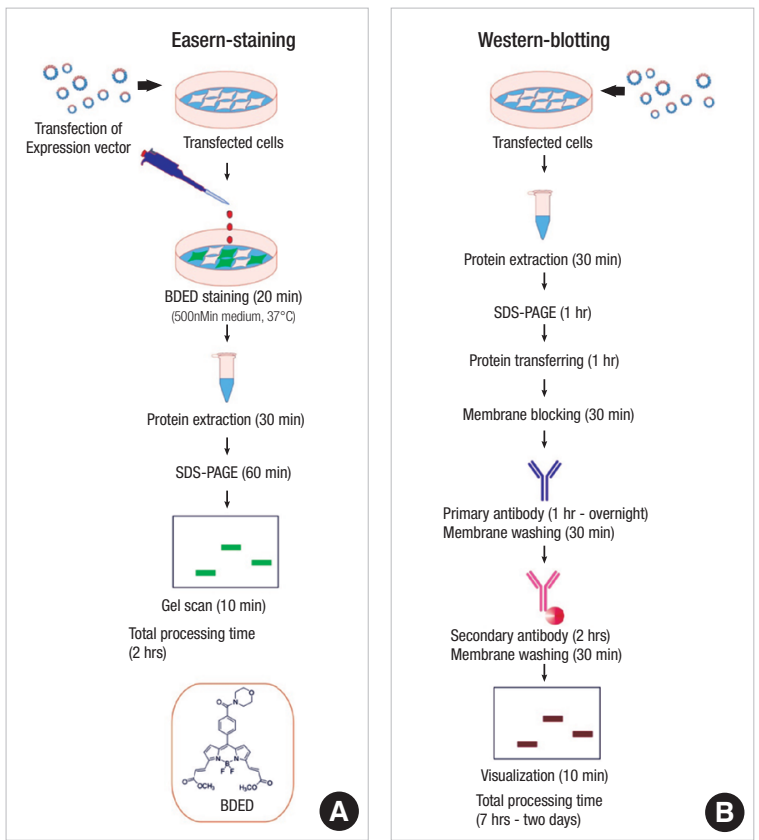

Key Words: Western blot; Estern staining; fluorescence dye BDED; RC-tag; peptide chemical tags 


\section{INTRODUCTION}

Recombinant protein expression and its detection is a crucial technique for biomedical research. Conventionally, denatured proteins in cell lysate are separated according to their molecular masses by electrophoresis, blotted to a membrane and detected by antibodies which are visualized by attached fluorophores or chemical reactions. Expression of the protein of interest is judged based on the size and antigenicity of the detected protein. This Western blotting technique is, however, highly dependent on the availability, affinity and specificity of antibodies which vary significantly from one to another. Small peptide tags such as c-myc, HA, FLAG, and polyhistidine have been introduced into recombinant proteins to simplify detection and purification of proteins ${ }^{1-4}$. Although the antibodies for the common tags are less variable compared to specific epitope-targetting antibodies, batch to batch variations of the antibody titre and quality are still inevitable.

Bioorthogonal chemistry, which covalently labels biomolecules with exogenously delivered chemical probes in a highly specific manner in living systems, has caught attention during last decade as a strategy for simple and easy labeling of proteins ${ }^{5}$. Due to the much smaller size of chemical probes compared to that of fulorescence proteins, which often perturbs the function of conjugated proteins because of bulky size, the function and structure of proteins are normally not affected by bioorthogonal labeling. The chemical labeling also overcomes the variation problem of antibodies descrived above. Among the switchable fluorescent chemical probes designed to become fluorescent by binding to small peptide tags are biarsenicals FlAsH-EDT 2 and ReAsH-EDT ${ }_{2}{ }^{6}$ and dimaleimide fluorogens ${ }^{7}$ which bind to 4 and 2 cysteine residues. We recently reported a novel bodipydiacrylate compound $4 \mathrm{~b}$ (BDED) and its binding peptide RCtag which has 2 cysteine residues placed next to arginines ${ }^{8}$. As the BDED compound is cell permeable and changes its emission spectrum from yellow to green by forming covalent bonds with the 2 cysteins in the RC-tag, both intracellular and extracelluar proteins can be labeled with visual confirmation of proper binding to the target. We have shown that RC-tagged exogenous protein labeled by BDED is conveniently visualized in SDS polyacrylamide gels gels by fluorescence scanning as well as in live cells. Using mCherry as a model protein we demonstrated RCtag's compatibility with other peptide tags and multimerization possibility to enhance fluorescence intensity.

Along with the remarkable development of fluorescencebased optical imaging technology, the use of small molecular weight fluorescent chemicals has been expanded in various fields of biomedical research. We expect that the need for visualization of proteins only with chemicals will increase superseding the use of fluorescence protein and antibody, particulary for live cell imaging, super-resolution microscopy and detection of recombinant protein. Despite its broad applicability and potential for dramatic increase of use, the chemical labeling technology for immediate visualization of protein has not been commonly termed. Therefore, we propose "Eastern staining" in reference to Southern blotting as a standard term for a recombinant protein detection method using fluorescent chimicals which bind to small peptide tags. As a proof of concept for an application of RC-tag in Eastern staining, we tagged 2 cytoskeletal proteins actin and tubilin and a cytosolic protein fatty acid binding protein 7 with a monomeric RC-tag in the current study. The expression of all the tagged proteins were conveniently confirmed by staining with BDED and fluorescence scanning of a SDS-poliacrylamide gel. As sumarized in Figure 1, the whole procedure of this method takes less than 2 hours while conventional Western-blotting takes at least 7 hours.

\section{RESULTS AND DISCUSSION}

\section{Evaluation of the applicability of Eastern staining on} various functional mammalian proteins

As a demonstration, we fused the $\mathrm{RC}$ tag to the $\mathrm{N}$-terminus of several functional proteins, such as human $\beta$-actin (42 kDa), human $\alpha$-tubulin (52 $\mathrm{kDa}$ ), and mouse fatty acid-binding protein 7 (Fabp7, 14 kDa) (Figure 2A). Recombinant proteins fused to RC tag together with c-myc tag were expressed in HEK 293A cells. After 48 hours, transfected cells were stained with BDED and the total cell extract was subjected to SDS-PAGE as depicted in Figure 1.

The size of green fluorescence bands appeared on the gel exactly corresponded to the expected molecular weight of respective proteins fused with tags (Figure 2B, left). The identities of the fluorescence bands were confirmed by the followed detection of c-myc tag by Western blotting (Figure 2B, middle). Perfect overlapping of the protein bands shown by BDED labeling and by c-myc tag Western blotting confirms the specificity and reliability of Eastern staining among the whole proteome (Figure 2B, right). Sensitivity of Eastern staining on RC-tag was comparable to that of c-myc Western-blotting by an anti c-myc antibody 9E10. Small amount of proteins, which are not distinguishable by Coomassie brilliant blue staining, are efficiently visualized by Eastern staining (Figure 2B, lanes 3, 4, 5).

\section{Application of Eastern staining in immunoprecipitation}

Eastern staining can be applied to various experiments, for example, immuno-precipitation (IP). IP is a widely used technique to isolate or enrich a specific protein from mixed proteins. The performance of IP is usually analyzed by Western-blotting using the respective antibodies against the proteins of interest. Therefore, small peptide tags such as c-myc tag, HA tag, or FLAG tag 


\section{Easern-staining}

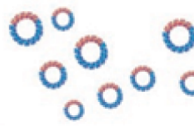

Transfection of Expression vector

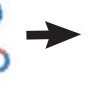

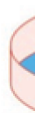

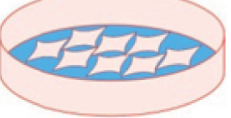

Transfected cells

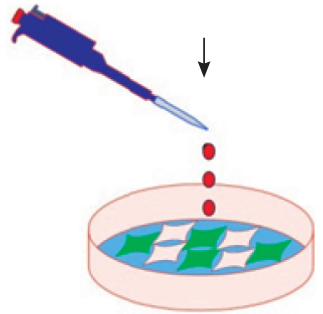

BDED staining (20 min) (500 nM in medium, $37^{\circ} \mathrm{C}$ )

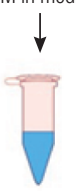

Protein extraction (30 min)

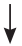

SDS-PAGE (60 min)

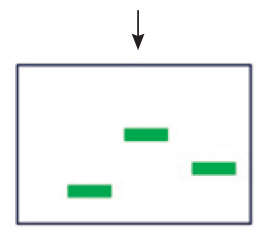

Gel scan (10 min)

Total processing time (2 hr)

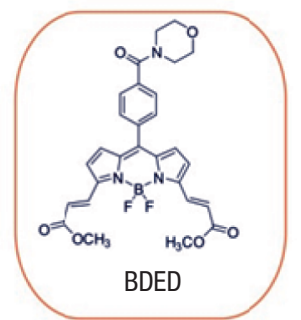

Western-blotting

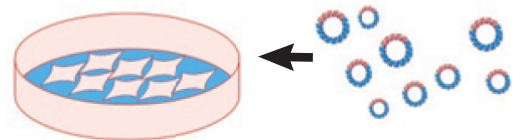

Transfected cells

$\downarrow$

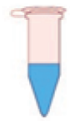

Protein extraction (30 min)

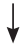

SDS-PAGE (1 hr)

$\downarrow$

Protein transferring (1 hr)

$\downarrow$

Membrane blocking (30 min)

$\downarrow$

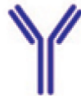

Primary antibody (1 hr - overnight) Membrane washing (30 min)

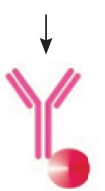

Secondary antibody (2 hr) Membrane washing (30 min)

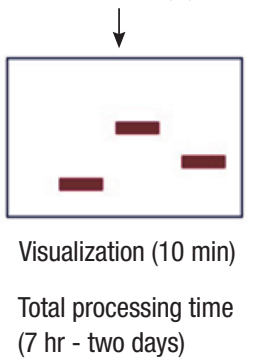

Figure 1. Time-saving procedure of RC-tag dependent "Eastern staining" (A) comparing to the conventional Western (immuno)-blotting (B) for the detection of recombinant protein.

are useful tools for IP of proteins for which the antibodies are not available. If the RC-tag is used in conjunction with those small tags, IP can be verified in a short processing time via Eastern staining. We immunoprecipitated hFabp7 fused with the cmyc tag and RC tag (RC $\cdot$ myc $\cdot$ Fabp 7$)$ using a monoclonal c-myc antibody (9E10) ${ }^{3}$. Transfected cells were stained with BDED and the total cell extract was subjected to IP followed by SDSPAGE. Eastern-staining facilitated the visualization of isolated proteins without laborious procedures of Western blotting, i.e., protein transfer to membrane, incubation with antibody, and multiple times of washing.

In Western blotting for immunoprecipiated proteins, the 


\begin{tabular}{l|l|l|l|l} 
& \multicolumn{2}{l}{$(2 \mathrm{kDa})$} & $(1.2 \mathrm{kDa})$ \\
RC myc Cherry & RC & myc & Cherry $(27 \mathrm{kDa})$ & $(30 \mathrm{kDa})$ \\
RC myc Action & RC & myc & $\beta$-action $(42 \mathrm{kDa})$ & $(45 \mathrm{kDa})$ \\
RC myc Tubulin & RC & myc & $\alpha$-tubulin $(52 \mathrm{kDa})$ & $(55 \mathrm{kDa})$ \\
RC myc Fabp7 & RC & myc & Fabp7 $(14 \mathrm{kDa})$ & $(17 \mathrm{kDa})$
\end{tabular}
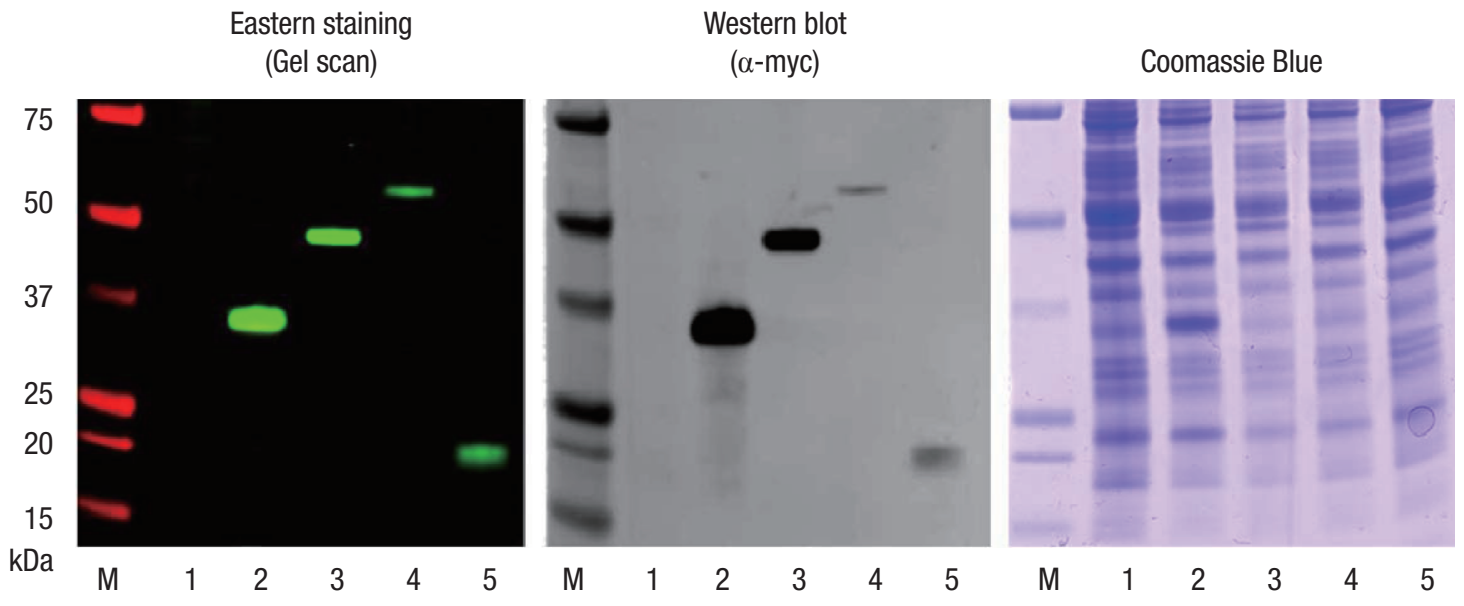

B

Figure 2. RC tag-mediated "Eastern staining" shows specific and reliable protein detection. (A) Chemical structure of BDED. (B) Schematic illustration for the RCtagged recombinant proteins. (C) Protein extracts from the cells, transfected with the vectors in B and stained with BDED, were analyzed in the SDS-PAGE and $\alpha$-myc Western blotting was followed to confirm the expression of tagged proteins. Coomassie brilliant blue staining data of the gel was prepared with equal amount of protein per lane for electrophoresis. M: protein size marker. N: no transfection (Gel scan Ex/Em = 488/SP 526 nm)

heavy chain and light chain of antibody are also detected as shown in Figure 3B (right), if the antibodies for Western blotting and IP are from the same species. It can make it difficult to detect the proteins when their sizes are similar with those of antibody chains. However, Eastern stanining is free from such unnecessary bands resulted from the antibodies (middle). The other experiments to study protein-protein interactions, such as "Co-IP" (protein complex-immuno-precipitation) or "pulldown assay" would be benefited by the Eastern staining?.

\section{Combination of RC tag with His-tag to detect the} expression of His-tagged protein in crude cell extract by Eastern staining

As an other example, we combined an RC-tag with a His-tag which is prevalently used in the affinity-purification of recombinant proteins expressed in diverse biological systems such as E. coli, yeast, insect cells, or mammalian cells ${ }^{10}$. Purification is performed by a time-consuming procedure employing the costly nickel column. Therefore, the expression of the His-tagged proteins in the biological system should be confirmed before conducting the affinity purification in a large scale. When a target protein is favorabley expressed in $E$. coli, the recombinant protein induced by the lac operon takes up a significant amount in total proteome profile ${ }^{11}$. Therefore, His-tagged proteins are obviously observed by staining the total proteome resolved in a gel by Coomassie brillaint blue or silver staining in our empirical observations.More sensitive methods, however, are required when the target proteins are produced in eukaryotic cells. Besieds Westrn-blotting using antibody against His-tag, different types of reporer agents, e.g., fluorophores, horse radish peroxidase (HRP), or alkaline peroxidase (AP), conjugated to nickelnitrilotriacetic acid (NTA) are developed for faster and easier detection $^{12,13}$. While those His-tag affinity probes are convenient to use, they are hardly applicable to the detection of small amount of His-tag fusion proteins since the transeint binding between the metal ion and imidazole ring lacks in sensitivity and stability. We thus combined an RC-tag to a His-tagged protein and conducted Eastern staining for the convenient in-process detection of proteins. His-tag was additionally fused to the RCtagged Fabp7, and the recombinant Fabp7 (RC $\cdot$ myc $\cdot$ Fabp $7 \cdot$ His) was expressed in mammalian cells. Small amount $(10 \mu \mathrm{g})$ of total proteins extracted from the transfected cells were incubated with BDED in vitro at room temperature. When the incubated total lysate was resolved in SDS-PAGE, His-tagged Fabp7 was 


\section{Easern staining in IP}

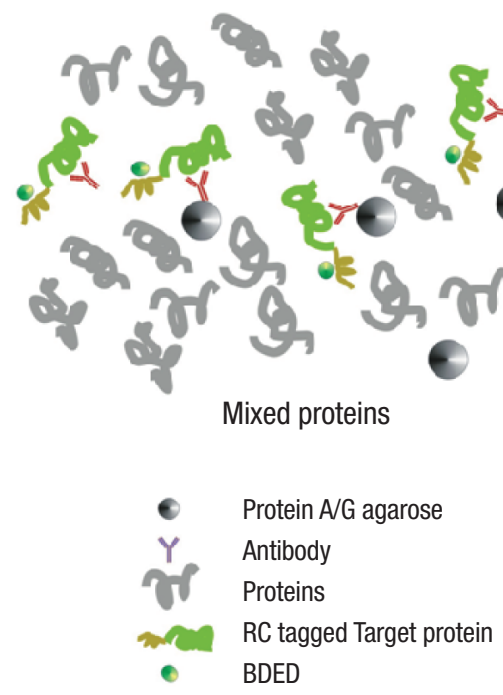

Before IP
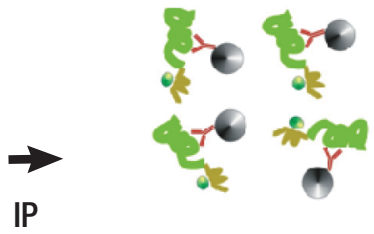

$\Downarrow$ SDS-PAGE

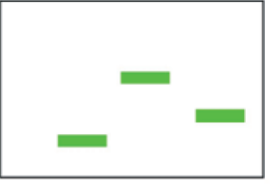

Gel scan

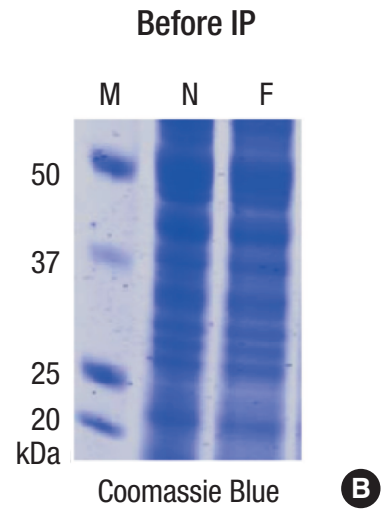

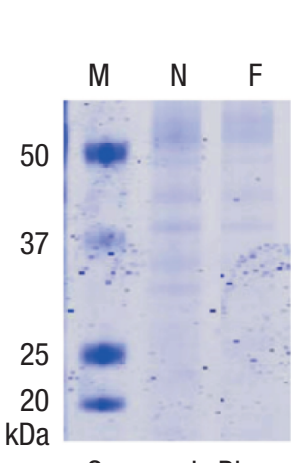

Coomassie Blue

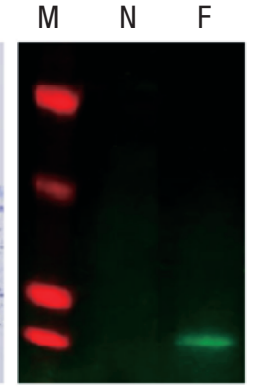

Gel scan

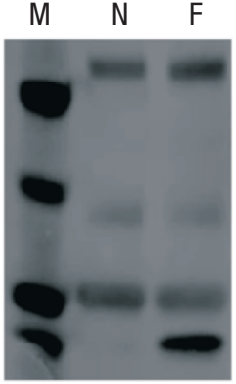

Western: $\alpha \mathrm{Myc}$ $\lg G(H)$

$\lg \mathrm{G}(\mathrm{L})$

Fabp7

\section{C}

Figure 3. Visulaization of immuno-precipitated protein by Eastern taining. RC $\cdot$ myc $\cdot$ Fabp7 was expressed in 293 A cells and the cells were stained with BDED before protein extraction. IP was performed with the 9E10 monoclonal antibody and the performance was revealed by "Eastern staining" and "Western (immuno)-blotting". (A) Schematic illustration of IP by using Eastern staining (B) Coomassie brilliant blue staining of total extract from 293A cells before IP. N: no transfection, F: pc-RC - myc · Fabp7 transfection. (C) Coomassie brilliant blue stainng after IP, Eastern staining by RC tag, and Western (immuno)-blotting by c-myc tag.

visualized due to the BDED bound to the RC-tag (Figure 4A). We compoared the sensitivity of RC-tag mediated Easterning staining with that of a commercial dye for His-tag fusion proteins, InVision ${ }^{\mathrm{TM}}$ His-tag In-gel Stain (Invitrogen). A fluorescent dye, conjugated to $\mathrm{Ni}^{2+}$-NTA, was applied directly to the protein gel after SDS-PAGE. As shown in Figure 4B, the affinity dye was able to visualize microgram quantity of fusion protein purified form E. coli protein extract (Figure 4B, lanes H-Fabp7 (5) and H-Fabp7 (10)), but the His-tagged Fabp7 in mammalina cell extract was not detectable.

We recommend to stain RC-tag with BDED in live cells for general Eastern staining of proteins in broad molecular weight range. When the total lysate of HEK293 cell was incubated with BDED in vitro, several protein bands of unknown identity were found at around $50 \mathrm{kDa}$ to $90 \mathrm{kDa}$. Therefore, in vitro Eastern staining of RC-tag would be applicable to the proteins at low molecular weight range (less than $50 \mathrm{kDa}$ ).

\section{CONCLUSION AND PROSPECTS}

Chemical tag is an emerging technology for convenient lableing of proteins in live cell. Since the pioneering invention of FlAsH/Tc-tag system by Tsien et al. ${ }^{6}$, diverse chemical tags have been competitively developed with different properties and advantages. Enzyme tags such as HaloTag or SNAP tag provide high specificity with minimal background, ${ }^{14,15}$ short peptide tags such as AviTag or LAP provide specific labeling by using transacting ligases, ${ }^{16,17}$ and self-affinity peptide tags such as Tc-tag and RC-tag ${ }^{6,8}$ enable minimally invasive tagging and intracellular staining with cell-permeable dyes. 

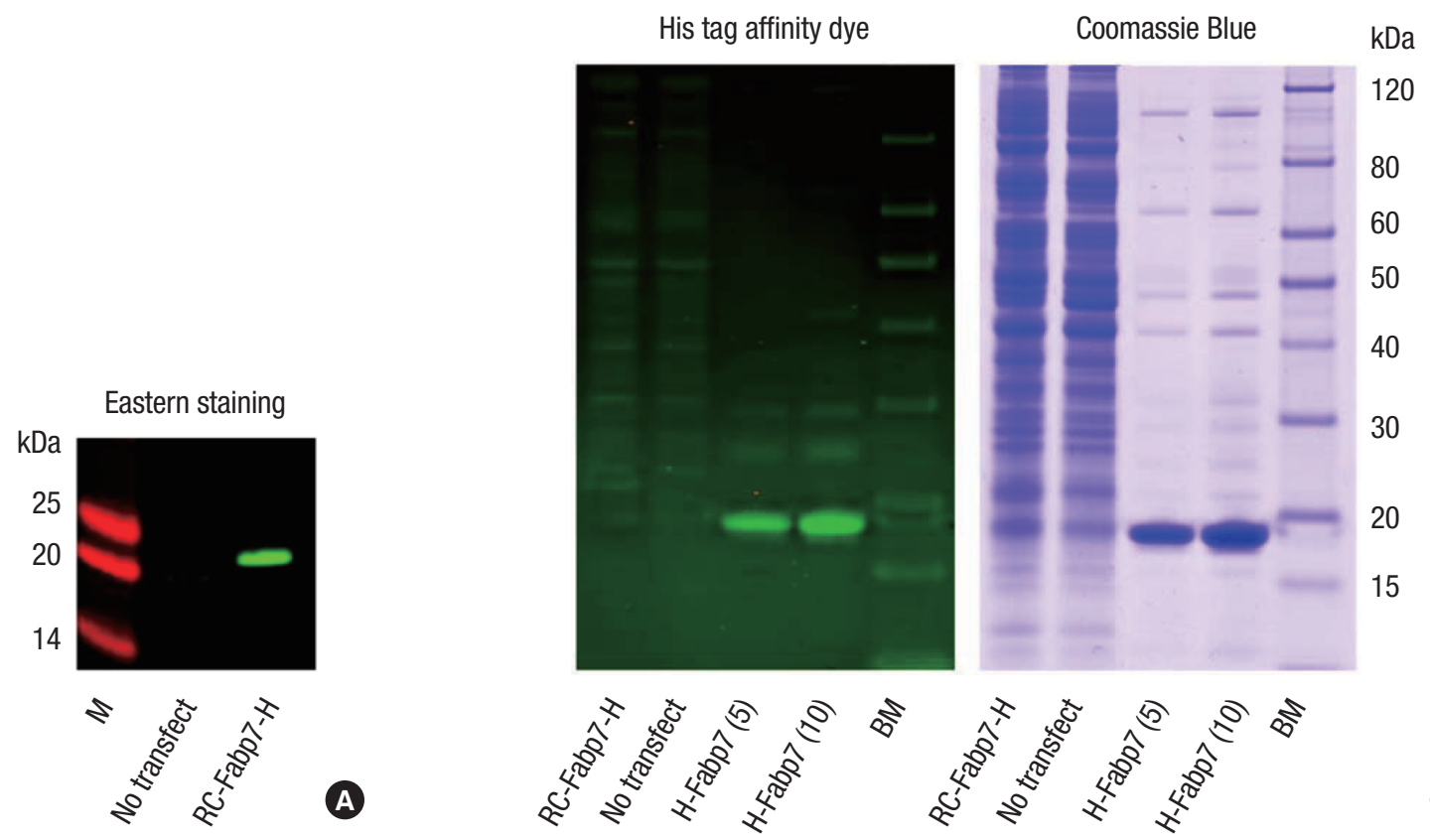

Figure 4. Eastern staining performed in in vitro provided convenient confirmation of recombinant protein expression. (A) Total protein (10 $\mu \mathrm{g})$ from the $293 \mathrm{~A}$ cells transfected with the pc-RC · myc · Fabp7 · His was incubated with BDED $(1 \mu \mathrm{M}, 15 \mathrm{~min}$, RT) and analyzed in SDS-PAGE. M: protein size marker, (B) $293 \mathrm{~A}$ cells were transfected with $\mathrm{pc}-\mathrm{RC} \cdot \mathrm{myc} \cdot \mathrm{Fabp} 7 \cdot$ His and the total protein extracts was resolved in SDS-PAGE. The gel was visualized on a fluorescence gel scanner after staining by using the InVision ${ }^{\mathrm{TM}}$ His-tag In-gel staining kit (Invitrogen) (left) and stained with Coomassie Brilliant Blue (right). Purified His-tagged Fabp7, produced in E. coli, was included as a positive control for the His-tagged protein. H-Fabp7 (5) and H-Fabp7(10) mean $5 \mu \mathrm{g}$ and $10 \mu \mathrm{g}$ of purified recombinant Fabp7, respectively. BM is the BenchMark ${ }^{\mathrm{TM}}$ His-tagged Protein Standard (Invitrogen) as a molecular weight marker.

Although most tags for chemical labeling were developed to facilitate optical imaging of proteins by circumventing the drawbacks of flourescent proteins, application of those tags can be expanded much further for various purposes. In this report, we introduced "Eastern staining using RC-tag and its binding fluorescence dye BDED" as a convenient protein detection method with examples of its application in biological experiments. This chemical tag-mediated protein detection method will significantly simplify a lot of experimental protocols which have required multi-step immuno-staining.

\section{MATERIALS AND METHODS}

\section{Expression vectors}

$\mathrm{pc}-\mathrm{RC} \cdot \mathrm{myc} \cdot$ Cherry was described in the previous report. ${ }^{8} p c$ $R C \cdot m y c \cdot$ Fabp 7: cDNA of mouse Fabp7 was prepared by RTPCR using the total RNA of NS5, a mouse neural stem cell line. The open reading frame (ORF) of Fabp7 was PCR-amplified with sense and antisense primers and the resulting PCR product was cloned into the BamHI/XhoI sites of pc-RC - myc which encodes the RC tag, together with the c-myc tag. This clone was named as $\mathrm{pc}-\mathrm{RC} \cdot \mathrm{myc}$

\section{- Fabp7.}

Fabp7 S_1: CCCGGATCCATGGTAGATGCTTTCTGCGC

\section{Fabp7 AS_1: CCCCTCGAGTGCCTTTTCATAACAGCGAA- CAGCA}

(Underlined are the restriction enzyme sites inserted for cloning).

$p c-R C \cdot m y c \cdot$ Actin and $p c-R C \cdot m y c \cdot$ Tubulin: DNA fragment encoding RC $\cdot$ myc was prepared by digestion of the pc-RC $\cdot$ myc with NheI/BamH1, separated by electrophoresis and eluted from the gel. Purified DNA fragment was cloned into the NheI/ BglII sites of pAcGFP1-Actin or pAcGFP1-Tubulin (Clontech) to exchange the GFP ORF with the DNA fragment encoding $\mathrm{RC} \cdot \mathrm{myc}$.

$p c-R C \cdot m y c \cdot F a b p 7 \cdot$ His: Fabp7 primers_2 were used to PCRamplify the expression cassette of Fabp7 with His tag at the Cterminus. The produced PCR product was digested with Bam$\mathrm{HI} / \mathrm{XhoI}$ and inserted into the BamHI/XhoI site of pc-RC $\cdot$ myc.

\section{Fabp7 S_2: CCCGGATCCATGGTAGATGCTTTCTGCG- CAACCT}

Fabp7 AS_2: CCCCTCGAGTCAATGGTGATGGTGATGATGTCTAGATGCCTTTCATAACAGCGAACAGCA

pQE30-Fabp7: PCR-amplified ORF of Fabp7 was digested with BamH1/XhoI and inserted into the BamH1/SalI sites of 
pQE30 (Qiagen) for the expression of N-terminal His-tagged Fabp7 in E. coil.

\section{Fabp7 S_1: CCCGGATCCATGGTAGATGCTTTCTGCGC Fabp7 AS_1: CCCCTCGAGTGCCTTTTCATAACAGCGAA- CAGCA}

Expression vectors used are pc-RC $\cdot \mathrm{myc} \cdot$ Cherry, pc-RC . myc $\cdot$ Fabp $7, \mathrm{pc}-\mathrm{RC} \cdot \mathrm{myc} \cdot$ Actin, pc-RC $\cdot$ myc $\cdot$ Tubulin, and pQE30-Fabp7. The generated clones were confirmed by DNA sequencing and purified by Qiagen Midi-prep column for transfection.

\section{Cell culture and transfection}

HEK293A cells, an immortalized line of primary human embryonic kidney cells, were purchased from Invitrogen and maintained in DMEM containing $10 \%$ fetal bovine serum, $1 \%$ antibiotics-antimycotics reagent. Cell culture materials were purchased from Invitrogen. For transient transfection, cells were plated at a density of $2 \times 10^{5}$ cells/well in 12 well plates and 500 ng of plasmid DNA were transfected with Lipofectamine 2,000 (Invitrogen). After incubation for two days, the transfected cells were harvested for experiments.

\section{Eastern staining}

BDED was reconstituted in DMSO as $1 \mathrm{mM}$ stock solution, and stored at $-20^{\circ} \mathrm{C}$. Immediately before staining, medium in the wells were drained and the BDED diluted in the pre-warmed serum-free medium was added directly onto the cells. After incubation $\left(20 \mathrm{~min}, 37^{\circ} \mathrm{C}\right)$, cells were washed with PBS and subjected to SDS-PAGE, IP, or Western-blotting.

\section{SDS-PAGE, gel-scanning, Western blotting, and}

Coomassie Brilliant Blue staining

Total protein was extracted by using CelLyticM ${ }^{\mathrm{TM}}$ cell lysis solution (Sigma). Generally $10 \mu \mathrm{g}$ of the protein/ well was loaded in SDS-PAGE gel for gel-scanning. NuPAGE Novex Bis-Tris Gels (Invitrogen) were used for PAGE and the gel was scanned in Typhoon 9410 Gel Scanner (GE Healthcare) using a $488 \mathrm{~nm}$ laser and and 526SP emission filter for the RC-tag: BDED observation. After gel scanning, proteins were transferred onto PVDF membrane for Western blotting. The mouse monoclonal anti cmyc (clone 9E10., Santa Cruz. Sc-40) antibody was visualized by secondary goat anti-mouse IgG conjugated with Cy5 (Invitrogen, A10524) which was detected by a $633 \mathrm{~nm}$ laser and 670BP emission filter on the Typhoon $9410 \mathrm{Gel} \mathrm{Scanner.} \mathrm{For} \mathrm{Coomass-}$ ie brilliant blue staining, the gel was stained in a staining solution $(50 \% \mathrm{MeOH}, 40 \%$ water, $10 \%$ glacial acetic acid, $0.25 \%$ Coomassie Brillaint Blue R-250) for 2-4 hours. Then, the background staining was removed by washing in destaining solu- tion ( $50 \% \mathrm{MeOH}, 40 \%$ water, $10 \%$ glacial acetic acid) by changing the destaining solution several times.

\section{Recombinant His-Fabp7 preparation}

pQE30-Fabp7 was transformed into E. coli strain SG13009 (Qiagen), which provides very stringent control of protein induction. Single colony of SG13009 (pQE30-Fabp7) was inoculated in $5 \mathrm{~mL}$ of LB medium containing ampicilline $(100 \mu \mathrm{g} / \mathrm{mL})$ and cultured for overnight. On the following day, $1 \mathrm{~mL}$ of the seed culture was put in $250 \mathrm{~mL}$ of LB medium and incubated until the optical density reaches 0.6 at $600 \mathrm{~nm}$ (OD600). Isopropyl $\beta$-D-1 thiogalactopyranoside (IPTG) was added to the medium at the working concentration of $0.5 \mathrm{mg} / \mathrm{mL}$ and recombinant protein was induced for 5 hours at $30^{\circ} \mathrm{C}$. Cells were harvested by centrifugation and the His-tagged Fabp7 was purified using the QIAexpress kit (Qiagen) according to the user manual. Purify of the recombinant protein was checked by SDS-PAGE and Coomassie brilliant blue staining.

\section{Immuno-precipitation}

Mammalian cell expression vector $\mathrm{pc}-\mathrm{RC} \cdot \mathrm{myc} \cdot \mathrm{Fabp} 7$ was transfected into 293A cells. After two days incubation, cells were stained with BDED and $100 \mu \mathrm{g}$ total lysate, prepared by using CelLyticM ${ }^{\mathrm{TM}}$ (Sigma), was incubated with 200 ng of monoclonal c-myc antibody (9E10) for overnight at $4^{\circ} \mathrm{C}$. On the following day, $20 \mu \mathrm{L}$ of Protein G-conjugated Sepharose beads (Pierce) were added to each of the lysate-antibody mixtures and incubated for 4 hours at $4^{\circ} \mathrm{C}$ with agitation. After three times repeated centrifugation and washing, $100 \mu \mathrm{L}$ of $1 \times$ LDS gel loading buffer (Invitrogen) was added to the beads and heated at $70^{\circ} \mathrm{C}$ for protein denaturation. IP samples were resolved in SDS-PAGE and the gel was observed on the gel scanner. After scanning, proteins in gel were transferred to PVDF membrane and Western blotting was performed against myc tag using the 9E10 antibody. The protein samples before and after immunoprecipitation were compared by SDS-PAGE and Coomassie brilliant blue staining (Figure 3).

\section{In vitro Eastern staining of His-tagged Fabp7 and} comparison with the InVision ${ }^{\mathrm{TM}}$ His-tag In-gel staining kit His-tagged Fabp7 was expressed in 293A cells by transfecting the pc-RC $\cdot$ myc $\cdot$ Fabp7 $\cdot$ His.Total cell lysates $(10 \mu \mathrm{g})$ from transfected and non-transfected cells were incubated with $10 \mu \mathrm{M}$ of BDED in tubes for 10 minutes at room temperature. After incubation, equal volume of $2 \times$ LDS gel loading buffer was added and the mixture was boiled. Denatured protein samples were resolved in SDS-PAGE and the gel was scanned (Figure 4A).

In the meantime, plasmids pc-RC $\cdot \mathrm{myc} \cdot \mathrm{Fabp} 7$ and pc$\mathrm{RC} \cdot \mathrm{myc} \cdot \mathrm{Fabp} 7 \cdot$ His were transfected into $293 \mathrm{~A}$ cells, and 10 $\mu \mathrm{L}$ of total cell lysates from each sample were resolved in SDS- 
PAGE. Equal amount of lysate from non-transfected cells and purified His-Fabp7 prepared by bacterial expression was loaded together for negative and positive control, respectively. After SDS-PAGE, the gel was stained with the InVision ${ }^{\mathrm{TM}}$ His-tag Ingel staining kit (Invitrogen) according to the manufacturer's protocol and scanned by a $532 \mathrm{~nm}$ laser and $560 \mathrm{~nm}$ long pass filter on the Typhoon $9410 \mathrm{Gel}$ Scanner. Immediately after scanning, the gel was stained with Coomassie brilliant blue (Figure 4B).

\section{Protocol for Eastern staining}

\section{A. Transfection}

i) HEK293A cells were maintained in DMEM (Invitrogen, cat. no. 11960) with $10 \%$ fetal bovine serum (Invtrogen, cat. no. 10099-141), 1\% penicillin/streptomycin (Invitrogen, cat. no. 15140-122).

ii) Cells in $10 \mathrm{~cm}$ plates were washed with PBS three times after aspirating the culture medium.

iii) Cells were incubating with $1 \mathrm{~mL}$ of Trypsin/1 mM EDTA solution (Invitrogen, cat. no. 25200-056) for 3 minutes and adding $2 \mathrm{~mL}$ of culture medium for neutralizing trypsin.

iv) Medium with cells were transferred to $15 \mathrm{~mL}$ conical tube. Supernatant was removing after centrifugation at 1,500 rpm during 3 minutes and $10 \mathrm{~mL}$ of fresh culture medium were re-suspending with pellets.

v) Cells were plated at a density of $2 \times 10^{5}$ cells/well in 12 well plates.

vi) Next day, $500 \mathrm{ng}$ of DNA was mixing with Lipofectamine 2000 (Invitrogen, cat. no. 11668-019) for 30 minutes in $1 \mathrm{~mL}$ of Opti-MEM I reduced-serum medium (Invitrogen, cat. no. 31985-062)

vii) HEK293A were incubating with Opti-MEM I reduced-serum medium containing DNA and lipofectamine 2000 for 2 days.

\section{B. Staining}

i) BDED was dissolved in DMSO (Sigma, cat. no. D2650) and kept at $-20^{\circ} \mathrm{C}$ as $1 \mathrm{mM}$ stock. Culture medium in the wells was exchanged to fresh medium including $500 \mathrm{nM}$ of BDED.

ii) After incubation with $500 \mathrm{nM} \mathrm{BDED}$ at $37^{\circ} \mathrm{C}$ for 20 minutes, cells were washing with PBS three times.

\section{Protein extraction}

i) Cells were incubating with CelLyticMTM cell lysis solution (Sigma, cat. no. C29780) including protease inhibitor cocktail (Roche, cat. no. 11836170001 ) at room temperature for 2 minutes.

ii) Cell lysates were collecting into $1.5 \mathrm{~mL}$ tube and supernatants were transferred to new $1.5 \mathrm{~mL}$ tube after centrifuga- tion at 14,000 rpm for 10 minutes.

iii) Concentration of total proteins was decided by Brad ford assay method. $10 \mu \mathrm{g}$ of the total proteins were mixed with Laemmli Sample Buffer (Bio-rad, cat. no. 161-0737) and boiled at $100^{\circ} \mathrm{C}$ for 5 minute.

iv) Boiled samples were loaded in NuPAGE Novex Bis-Tris Gels (Invitrogen) and run under $200 \mathrm{~V}$ for 30 minutes for fractionating total proteins.

\section{Visualization}

i) Gel was scanned in Typhoon $9410 \mathrm{Gel}$ Scanner (GE Healthcare) using a $488 \mathrm{~nm}$ laser and 526SP emission filter for visualizing of the RC-tag: BDED signals.

\section{E. Confirmation of protein expression}

After scanning, proteins were transferred onto PVDF membrane by wet transfer device (Bio-rad, cat. no. 165-8029) for checking expressed proteins. PVDF membrane was incubating with $1 \mathrm{X}$ TTBS solution including 5\% skim milk. Finally, protein was visualized after incubating with mouse monoclonal anti c-myc (clone 9E10., Santa Cruz. Sc-40) antibody and secondary goat anti-mouse IgG conjugated with Cy5 (Invitrogen, A10524) which was detected by $633 \mathrm{~nm}$ laser and 670BP

\section{ACKNOWLEDGEMENTS}

This work was supported by the intramural funding from the A*STAR Biomedical Research Council and a Singapore Ministry of Education Academic Research Fund Tier 2 (MOE2010T2-1-025).

\section{REFERNCES}

1. Brizzard, B. (2008). Epitope tagging. BioTechniques 44, 693-695.

2. Terpe, K. (2003). Overview of tag protein fusions: from molecular and biochemical fundamentals to commercial systems. Appl Microbiol Biotechnol 60, 523-533.

3. Evan, G.I., Lewis, G.K., Ramsay, G., and Bishop, J.M. (1985). Isolation of monoclonal antibodies specific for human c-myc proto-oncogene product. Mol Cell Biol 5, 3610-3616.

4. Einhauer, A., and Jungbauer, A. (2001). The FLAG peptide, a versatile fusion tag for the purification of recombinant proteins. J Biochem Biophys Meth 49, 455-465.

5. Prescher, J.A., and Bertozzi, C.R. (2005). Chemistry in living systems. Nat Chem Biol 1, 13-21.

6. Griffin, B.A., Adams, S.R., and Tsien, R.Y. (1998). Specific covalent labeling of recombinant protein molecules inside live cells. Science 281, 269-272.

7. Girouard, S., Houle, M.H., Grandbois, A., Keillor, J.W., and Michnick, S.W. (2005). Synthesis and characterization of dimaleimide fluorogens designed for specific labeling of proteins. J Am Chem Soc 127, 559-566. 
8. Lee, J.J., Lee, S.C., Zhai, D., Ahn, Y.H., Yeo, H.Y., Tan, Y.L., and Chang, Y.T. (2011). Bodipy-diacrylate imaging probes for targeted proteins inside live cells. Chem Commun (Camb) 47, 4508-4510.

9. Einarson, M.B. (2001). In Molecular Cloning: A Laboratory Manual, 3rd Edition (Cold Spring Harbor Kaboratory Press).

10. Hengen, P. (1995). Purification of His-Tag fusion proteins from Escherichia coli. Trends Biochem Sci 20, 285-286.

11. Donovan, R.S., Robinson, C.W., and Glick, B.R. (1996). Review: optimizing inducer and culture conditions for expression of foreign proteins under the control of the lac promoter. J Ind Microbiol 16, 145-154.

12. Krishnan, B., Szymanska, A., and Gierasch, L.M. (2007). Site-specific fluorescent labeling of poly-histidine sequences using a metal-chelating cysteine. Chem Biol Drug Des 69, 31-40.

13. Zhao, C., Hellman, L.M., Zhan, X., Bowman, W.S., Whiteheart, S.W., and Fried, M.G. (2010). Hexahistidine-tag-specific optical probes for analyses of proteins and their interactions. Anal Biochem 399, 237-245. 14. Los, G.V., Encell, L.P., McDougall, M.G., Hartzell, D.D., Karassina, N., Zimprich, C., Wood, M.G., Learish, R., Ohana, R.F., Urh, M., et al. (2008). HaloTag: a novel protein labeling technology for cell imaging and protein analysis. ACS Chem Biol 3, 373-382.

15. Keppler, A., Gendreizig, S., Gronemeyer, T., Pick, H., Vogel, H., and Johnsson, K. (2003). A general method for the covalent labeling of fusion proteins with small molecules in vivo. Nat Biotechnol 21, 86-89.

16. Chen, I., Howarth, M., Lin, W., and Ting, A.Y. (2005). Site-specific labeling of cell surface proteins with biophysical probes using biotin ligase. Nat Methods 2, 99-104.

17. Fernandez-Suarez, M., Baruah, H., Martinez-Hernandez, L., Xie, K.T., Baskin, J.M., Bertozzi, C.R., and Ting, A.Y. (2007). Redirecting lipoic acid ligase for cell surface protein labeling with small-molecule probes. Nat Biotechnol 25, 1483-1487. 